ARTICLE

Received 12 Sep 2015 | Accepted 26 Oct 2015 | Published 8 Dec 2015

DOI: $10.1038 /$ ncomms10041

OPEN

\title{
Asymmetric intramolecular $\alpha$-cyclopropanation of aldehydes using a donor/acceptor carbene mimetic
}

Chaosheng Luo ${ }^{1}$, Zhen Wang ${ }^{1} \&$ Yong Huang ${ }^{1}$

Enantioselective $\alpha$-alkylation of carbonyl is considered as one of the most important processes for asymmetric synthesis. Common alkylation agents, that is, alkyl halides, are notorious substrates for both Lewis acids and organocatalysts. Recently, olefins emerged as a benign alkylating species via photo/radical mechanisms. However, examples of enantioselective alkylation of aldehydes/ketones are scarce and direct asymmetric dialkylation remains elusive. Here we report an intramolecular $\alpha$-cyclopropanation reaction of olefinic aldehydes to form chiral cyclopropane aldehydes. We demonstrate that an $\alpha$-iodo aldehyde can function as a donor/acceptor carbene equivalent, which engages in a formal $[2+1]$ annulation with a tethered double bond. Privileged bicyclo[3.1.0]hexane-type scaffolds are prepared in good optical purity using a chiral amine. The synthetic utility of the products is demonstrated by versatile transformations of the bridgehead formyl functionality. We expect the concept of using $\alpha$-iodo iminium as a donor/acceptor carbene surrogate will find wide applications in chemical reaction development.

\footnotetext{
${ }^{1}$ Key Laboratory of Chemical Genomics, Shenzhen Graduate School, Peking University, Xili University Town, Shenzhen 518055, China. Correspondence and requests for materials should be addressed to Y.H. (email: huangyong@pkusz.edu.cn).
} 
A symmetric $\alpha$-alkylation of carbonyl compounds plays a vital role in organic synthesis ${ }^{1}$. It was traditionally accomplished via chiral enolate chemistry ${ }^{2-4}$. Modified carbonyls, such as tin enolates and acidic imino esters, have been successfully alkylated under Lewis acid or phase transfer catalysis $^{5-7}$. However, the more useful direct $\alpha$-alkylation of aldehyde/ketone remains unsolved. Progress in this area benefited from the recent advance of enamine organocatalysis. Limited success was received using alkyl halides or stabilized carbocation precursors $^{8-19}$. Practically, alkyl halides are not well tolerated by either Lewis acids or nucleophilic organocatalysts because of their strong electrophilicity and interference by forming strong hydrogen halide by-products. Recently, olefins became a viable alkylation agent under mild redox catalysis ${ }^{20,21}$.

Cyclopropane is a preferred structural motif for medicinal chemistry, owing to its rigidity and metabolic stability ${ }^{22}$. In particular, ring-fused cyclopropanes are prevalent among bioactive natural products and drug molecules. In sharp contrast to their biological stability, fused cyclopropanes carry unique chemical reactivity for fragmentation and rearrangement that is often exploited in natural product synthesis ${ }^{23-25}$. To access these structurally unique scaffolds, a number of synthetic methods have been developed. Typically, these compounds were made via an annulation reaction between an olefin and a tethering one carbon donor/acceptor such as metal carbenoids ${ }^{26-38}$. Nevertheless, asymmetric synthesis of bicyclic cyclopropanes remains very challenging $27,29,37$.

Since the renaissance of enamine-iminium catalysis in 2000 (refs 39,40), enantioselective $\alpha$-functionalization of carbonyls has taken the centre stage for synthetic innovation. Despite tremendous advance in this field, examples of direct amine catalysed $\alpha$-alkylation of aldehydes are rare. Among them, two intramolecular $\alpha$-alkylation strategies are notable: in 2004, Vignola and List reported the first enamine catalysed intramolecular alkylation using halo aldehydes ${ }^{8}$. Using this strategy, a cyclopropane aldehyde product was prepared. In 2013, MacMillan and co-workers accomplished intramolecular $\alpha$-alkylation of aldehydes having a tethering double bond via enamine-singly occupied molecular orbital catalysis ${ }^{41}$. Alternatively, both MacMillan and Córdova reported methods to generate $\alpha$-cyclopropane aldehydes from $\alpha, \beta$-unsaturated aldehydes using an iminium-enamine cascade strategy ${ }^{42-45}$. Overviewing recent progress of organocatalytic $\alpha$-alkylation reactions, efforts have been concentrated on replacing only one hydrogen atom at the $\alpha$ position of aldehydes with alkylating agents. In sharp contrast, double alkylation of the carbonyl $\alpha$-methylene has been largely neglected ${ }^{13}$.

Herein, we report an orthogonal approach for direct $\alpha$-cyclopropanation of aldehydes via intramolecular $\mathrm{C}=\mathrm{C} / \mathrm{CH}_{2}$ annulation, enabling rapid access to bicyclo[3.1.0]hexanes and their aza analogues (Fig. 1). This strategy uses an $\alpha$-iodo iminium species as a key donor/acceptor carbene mimetic.

\section{Results}

Rationale design of a new donor/acceptor carbene mimetic. Recently, we reported an $\alpha$-allenylation reaction of aldehyde using gold/amine synergistic catalysis, in which the $\alpha$-methylene of carbonyl was converted to an $\mathrm{sp}^{2}$ centre ${ }^{46}$. Along this line, we decided to explore the possibility of forming a cyclopropane through oxidative annulation between the aldehyde $\alpha-\mathrm{CH}_{2}$ and a tethering double bond. Conceptually, the net dehydrogenative nature of this transformation is counterintuitive. Normally, cyclopropanation of an olefin requires a donor/acceptor onecarbon counterpart such as a metal carbenoid, whereas the $\alpha$-carbon of aldehydes is nucleophilic (donor only). We envisioned that an $\alpha$-halo aldehyde intermediate might serve such a purpose. Upon mixing with a secondary amine, the corresponding $\alpha$-halo iminium becomes a highly electrophilic alkylation species, whereas its complementary enamine form remains nucleophilic. This donor/acceptor reactivity might be synthetically equivalent to a carbene (Fig. 2).

Initial attempts on intramolecular $\alpha$-cyclopropanation. Enantioselective $\alpha$-halogenation of aldehydes have been reported by several research groups ${ }^{47-56}$. Among those reported examples, chlorination of citronellal ${ }^{55,56}$ seemed suitable as the starting point of our investigation. Unfortunately, attempts to affect the cyclopropanation reaction using $\alpha$-chloro citronellal were fruitless. In the presence of a secondary amine, no cyclopropane aldehyde was detected. Further activation of the carbon-chloride bond using various Lewis acids resulted in substrate decomposition in most cases (Fig. 3). We speculated that chlorides are not electrophilic enough to react with an olefin, which is a rather poor close shell nucleophile. Next, we focused our attention on an iodination/annulation cascade. To further facilitate ring closure, we decided to install gem-diesters in the middle of the chain to assist conformational folding for intramolecular reaction (the Thorpe-Ingold effect).

We were excited to find out that treating aldehyde 1a with 1 equiv. of amine $3 \mathbf{a}$ (ref. 57) and $\mathrm{N}$-iodosuccinimide (NIS) yielded the corresponding bicyclo[3.1.0] hexane aldehyde 2a in moderate yield and enantiomeric ratio (er; Fig. 3). No reaction occurred in the absence of NIS. Interestingly, a catalytic amount of amine $\mathbf{3 a}$ failed to give any product. Careful examination of the crude reaction mixture revealed that prolinol ether 3a quickly decomposed to benzophenone and pyrrolidin-2one. Control experiment showed that mixing 3a with NIS resulted in full conversion to benzophenone within $5 \mathrm{~min}$ at room temperature. As a result, $\alpha$-iodination was undermined. The redox lability of amine $\mathbf{3 a}$ is likely a result of high electron density on the nitrogen atom, which might be attenuated using a weak Brønsted acid. Upon screening various acids, we found that $p$-nitrobenzoic acid ( $p$ NBA) stabilizes 3a under the reaction condition. When simple isopentaldehyde was subjected to the $\alpha$-iodination reaction, the product was obtained in $35 \%$ yield using 20 mol\% 3a, compared with a $90 \%$ conversion with 1 equiv. of 3a. Upon adding $p$ NBA (1 equiv.) as additive, $90 \%$ yield was obtained using $20 \mathrm{~mol} \% \mathbf{3 a}$ and no decomposition of the amine was observed.

Optimization of the NIS-mediated $\alpha$-cyclopropanation. Even though we were able to address the amine decomposition problem, the overall cyclopropanation cascade performed poorly using catalytic amine. Nevertheless, the use of $p \mathrm{NB} A$ continued to improve the efficiency of reactions employing 1 equiv. 3a. We next examined solvent effect. The reaction proceeds in a number of non-polar solvents (Table 1, entries 1-6). Good enantioselectivity was observed in toluene and ether. Various iodination reagents were also investigated. 1,3-Diiodo-5,5-dimethylhydantoin afforded a very similar result as NIS (Table 1, entry 7). Stronger $\mathrm{I}^{+}$reagents, such as $\mathrm{I}_{2}, \mathrm{ICl}, \mathrm{IBr}$, led to very low conversions (Table 1, entries 8-11). Substrate decomposition was predominant. The amine promoters were studied systematically. The MacMillan's imidazolidinone catalyst ${ }^{40}$ was not productive (Table 1, entry 16). 2,5-Diarylpyrrolidine $3 \mathrm{e}^{13 \mathrm{a}}$ afforded racemic $\mathbf{2 a}$ in moderate yield (Table 1, entry 15). Varying the size of the silicon group did not improve selectivity (Table 1, entries 12-14). The original catalyst $\mathbf{3 a}$ afforded the highest conversion. Although the 3,5-( $\left(\mathrm{CF}_{3}\right)_{2}-\mathrm{Ph}$ analogue $3 \mathrm{~g}$ led to higher er than 3a (90.5/9.5 versus 84/16; Table 1, entry 17 and entry 5), the yield 
a Classical $\alpha$-functionalization via enamine catalysis (numerous reports)

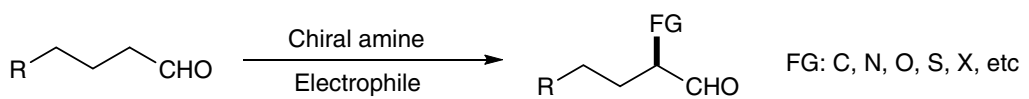

b Intramolecular alkylation via enamine catalysis<smiles>CCOCC(CO)(CC=O)COCC</smiles><smiles>CCOCCN1CCC[C@@]1(C)C(=O)O</smiles><smiles>CCOCC1(C(=O)OCC)C[C@H]1C=O</smiles>

List

$70 \%$, er $=93: 7$<smiles>CC(C)=CCCCCC=O</smiles><smiles>[R]CC1NC(C(C)(C)C)N(C)C1=O</smiles>

MacMillan

$62 \%$, er $=92.5: 7.5$

C Intramolecular $\alpha$-cyclopropanation of aldehydes promoted by a secondary amine (this work)

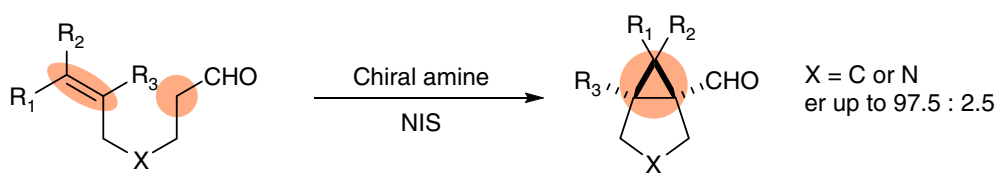

Figure 1 | $\alpha$-Functionalization of aldehydes via enamine organocatalysis. (a) A number of reactions dealing with enantioselective $\alpha$-functionalization of aldehydes have been reported using the classical enamine organocatalysis. (b) Two representative reports of intramolecular $\alpha$-alkylation of aldehydes are shown. (c) Our strategy for direct $\alpha$-cyclopropanation of aldehydes. DME, dimethoxyethane; FG, functional group; NIS, N-lodosuccinimide; phen, 1 , 10-phenanthroline; $\mathrm{Tf}_{2} \mathrm{~N},\left(\mathrm{CF}_{3} \mathrm{SO}_{2}\right)_{2} \mathrm{~N}$.

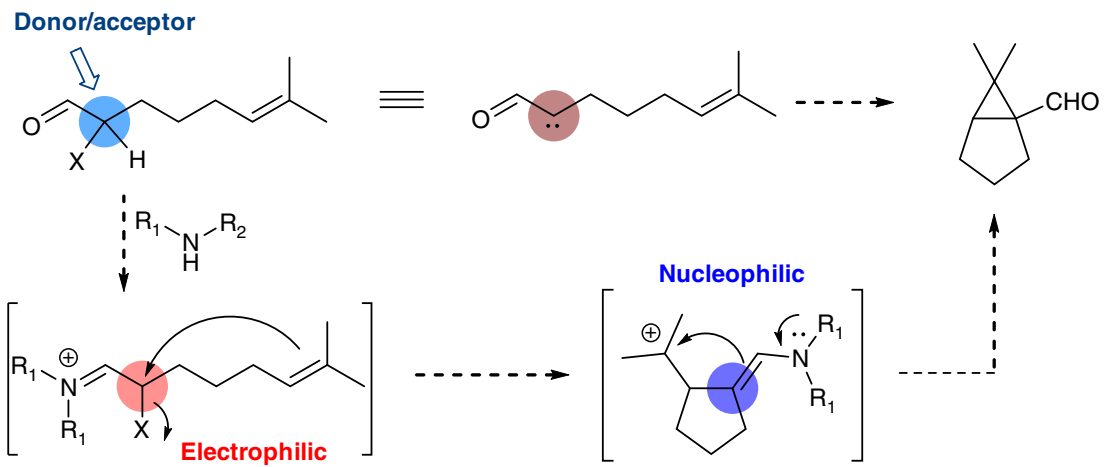

Figure 2 | Design of $\alpha$-halo iminium as a donor/acceptor carbene mimetic. We propose the $\alpha$-carbon of $\alpha$-halo aldehyde might serve as a donor/acceptor by the action of a secondary amine. In iminium form, the halide is a good electrophile. In enamine form, the carbon is a good nucleophile.

dropped substantially. We speculated that both the electronic and the steric nature of the aryl group play an important role: electron-neutral aryls lead to higher yields; bulky 3,5-disubstitution improves er. Following this rationale, we prepared the corresponding 3,5- $(t-\mathrm{Bu})_{2}$ amine 31. Improved yield was obtained at no cost of the selectivity (Table 1, entry 22). With this optimized chiral amine in hand, we quickly re-examined solvent effect. Further improvement of both yield and er was achieved using $i \operatorname{Pr}_{2} \mathrm{O}$ as the reaction media (Table 1, entry $24,75 \%$ yield, 93:7 er).

Substrate scope. The substrate scope was studied next (Table 2). The cyclopropanation occurs smoothly using either carbon or nitrogen as the linker between carbonyl and olefin. Only cis-bicyclic isomers were isolated because of ring strain control. However, gem-disubstitution is required for the all-carbon linkers, without which the reaction becomes very slow. Trialkyl substituted double bond affords highest yield and er. Exo-cyclic double bonds were well tolerated, leading to bicyclo[3.1.0]hexanes with an additional ring spiro to the cyclopropane (Table 2, products $\mathbf{2} \mathbf{f}-\mathbf{2 h}$ ). The selectivity of these substrates increases with the size of the ring. When a cyclic ketal was used as the linker, both high yield and excellent er were obtained (Table 2, product 2i). Interestingly, a 3-fuse-5-spiro-4 ring skeleton was obtained using an azetidine-containing substrate $\mathbf{1 j}$. The alkyl substituents at the double bond terminal is essential. Removing these groups led to low reactivity and poor er (Table 2 , products $\mathbf{2} \mathbf{k}$ and $\mathbf{2} \mathbf{l}$ ). 
a $\alpha$-Chloro aldehyde was not productive<smiles>CC(C)=CCC[C@@H](C)CC=O</smiles>

b Initial success using NIS<smiles>CC(C)=CCC(CCC=O)(C(=O)O)C(=O)OBr</smiles>

$1 a$

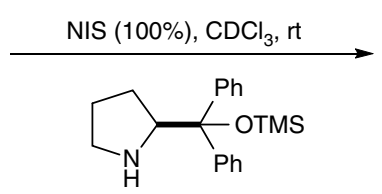

3a

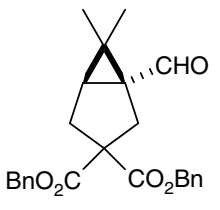

2a

C Interference between amine and NIS<smiles>COC(c1ccccc1)(c1ccccc1)C1CCCN1</smiles><smiles>CC[AsH2]</smiles><smiles>O=C(c1ccccc1)c1ccccc1</smiles>

Quant. yield

\begin{tabular}{l|l}
\hline $\mathbf{3 a}$ & $\mathbf{2 a}$ \\
\hline $20 \%$ & $0 \%$ \\
$100 \%$ & $73 \%$, er $=76: 24$ \\
\hline
\end{tabular}

d Effect of the acid additive<smiles>CC(C)CC=O</smiles><smiles>COC(c1ccccc1)(c1ccccc1)C1CCCN1</smiles><smiles>CC(C)C(I)C=O</smiles>

\begin{tabular}{ll}
\hline $\mathbf{3 a}$ & Product \\
\hline $100 \%$ & $90 \%$ \\
$20 \%$ & $35 \%$ \\
$20 \%+1$ equiv. pNBA & $90 \%$ \\
\hline
\end{tabular}

Figure 3 | Preliminary exploration of $\alpha$-halo aldehydes as the key intermediate. (a) $\alpha$-Chloro citronellal failed to undergo $\alpha$-cyclopropanation using various combinations of amines and Lewis acids. (b) The $\alpha$-cyclopropanation occurred smoothly for substrate 1a using 1 equiv. NIS and chiral amine $\mathbf{3 a}$. A catalytic amount of $\mathbf{3 a}$ did not promote this reaction. (c) Amine 3a was found to react with NIS rapidly at room temperature (rt). Benzophenone was found to be the major decomposition product. (d) Owing to serious decomposition of $\mathbf{3 a}$ by NIS, $\alpha$-iodination of isobutyraldehyde requires stoichiometric amine 3a. However, we found that para-nitrobenzoic acid ( $p N B A$ ) stabilizes this amine, allowing for catalytic use. er, enantiomeric ratio; TMS, trimethylsilyl. TFA, trifluoroacetic acid; NCS, N-Chlorosuccinimide; OTMS, trimethylsilyloxyl.

When a tetrasubstituted olefin was used, a fully substituted cyclopropane product with three contiguous all-carbon quaternary centres was obtained as the single cis-diastereomer in $71 \%$ yield and 91:9 er (Table 2, product $\mathbf{2 m}$ ). We also attempted intermolecular $\alpha$-cyclopropanation reactions using simple aldehydes and various electron-rich olefins. Unfortunately, no desired cyclopropane product was obtained under the standard reaction condition. The $\alpha$-iodo aldehyde intermediates were the major products. This result suggests a large entropy penalty is required for the key carbon-carbon bond formation.

Mechanistic experiments. To further understand the mechanism of this unconventional cascade cyclopropanation. We carried out real-time ${ }^{1} \mathrm{H}$ NMR experiments using deuterated solvent. The starting material was consumed within 10 min upon addition of NIS. The corresponding $\alpha$-iodo aldehyde $4 \mathbf{a}$ was obtained in quantitative yield, which was slowly converted to product 2 a. In fact, $\alpha$-iodo aldehyde $4 a$ could be isolated by quick flash column chromatography. Subjecting $\mathbf{4 a}$ to amine alone led to the cyclopropane product in good yield without the need of NIS. Therefore, $\alpha$-iodo aldehyde is very likely the intermediate for the overall cyclopropanation. It is noteworthy that the selectivity of the first step is poor. Er of $\mathbf{4 a}$ was 61:39 upon isolation after $5 \mathrm{~min}$. However, when $\mathbf{4 a}$ was isolated after $40 \mathrm{~min}$, it became racemic (Fig. 4a). This rapid decrease of optical purity for 4a indicated a facile racemization via enamine formation. Therefore, the stereoselectivity of the overall reaction is solely controlled in the annulation step. Er of the $\alpha$-iodo intermediate ought to be inconsequential for the ultimate selectivity of the bicyclic product. Indeed, when racemic $4 \mathbf{a}$ was subjected to catalyst 31 in $i \operatorname{Pr}_{2} \mathrm{O}$, 2a was isolated in $82 \%$ yield and 93:7 er (Fig. 4b).

The annulation step was also studied. We prepared $E / Z$ isomer pairs of disubstituted olefin $\mathbf{1 n}$. The trans-product $\mathbf{2 n}$ was obtained as the major isomer regardless of the $E / Z$ ratio of the starting material. Furthermore, a mixture (1.2:1) of diastereomers was obtained when the pure E-form of 10 was used (Fig. 5). Hence, the olefin/iodide annulation is a stepwise process, during which bond rotation occurs. Based on this observation, a free singlet carbene intermediate is highly unlikely. The cyclopropanation could occur through several pathways: (i) halogen extraction by the electron-rich olefin to form an iodiranium species, followed by enamine promoted cyclization and elimination of hydrogen iodide; (ii) double electrophilic alkylation pathway (first, Friedel-Crafts alkylation between olefin and iodide; second, alkylation of enamine by the resulting carbocation, Fig. 2); (iii) radical mechanism by homo-cleavage of the $\mathrm{C}-\mathrm{I}$ bond. We obtained the X-ray structure of $\alpha$-iodo aldehyde $4 c$. In solid phase, the double bond is oriented $180^{\circ}$ away from the iodine atom, an ideal alignment for $\mathrm{SN}_{2}$ displacement. Although we could not rule out the possibility of a radical cyclization, attempts to intercept the tertiary radical were unsuccessful. The 
Table 1 | Condition optimization.

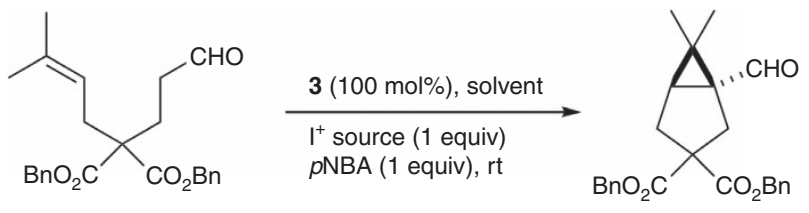

$1 a$

2a

\begin{tabular}{ll}
\hline Entry & Solvent \\
\hline 1 & THF \\
2 & DCM \\
3 & Acetone \\
4 & Et $_{2} \mathrm{O}$ \\
5 & Toluene \\
6 & Dioxane \\
7 & Toluene \\
8 & Toluene \\
9 & Toluene \\
10 & Toluene \\
11 & Toluene \\
12 & Toluene \\
13 & Toluene \\
14 & Toluene \\
15 & Toluene \\
16 & Toluene \\
17 & Toluene \\
18 & Toluene \\
19 & Toluene \\
20 & Toluene \\
21 & Toluene \\
22 & Toluene \\
23 & Et ${ }_{2} \mathrm{O}$ \\
24 & (i-Pr) ${ }_{2} \mathrm{O}$ \\
& \\
& \\
& \\
& \\
& \\
& H \\
& \\
&
\end{tabular}

I $^{+}$source
$\mathrm{NIS}$
$\mathrm{NIS}$
$\mathrm{NIS}$
$\mathrm{NIS}$
$\mathrm{NIS}$
$\mathrm{NIS}$
$\mathrm{DIH}$
$\mathrm{I}_{2}$
$\mathrm{Py} \mathrm{IBF}_{4}$
$\mathrm{ICl}$
$\mathrm{IBr}$
$\mathrm{NIS}$
$\mathrm{NIS}$
$\mathrm{NIS}$
$\mathrm{NIS}$
$\mathrm{NIS}$
$\mathrm{NIS}$
$\mathrm{NIS}$
$\mathrm{NIS}$
$\mathrm{NIS}$
$\mathrm{NIS}$
$\mathrm{NIS}$
$\mathrm{NIS}$
$\mathrm{NIS}$
$\mathrm{N}$
$\mathrm{H}$

$3 c$

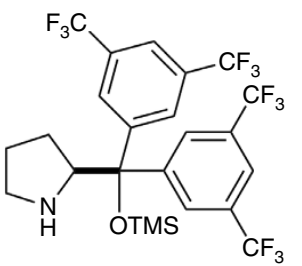

3g

\begin{tabular}{c} 
Amine \\
\hline $\mathbf{3 a}$ \\
$\mathbf{3 a}$ \\
$\mathbf{3 a}$ \\
$\mathbf{3 a}$ \\
$\mathbf{3 a}$ \\
$\mathbf{3 a}$ \\
$\mathbf{3 a}$ \\
$\mathbf{3 a}$ \\
$\mathbf{3 a}$ \\
$\mathbf{3 a}$ \\
$\mathbf{3 a}$ \\
$\mathbf{3 b}$ \\
$\mathbf{3 c}$ \\
$\mathbf{3 d}$ \\
$\mathbf{3 e}$ \\
$\mathbf{3}$ \\
$\mathbf{3 g}$ \\
$\mathbf{3 h}$ \\
$\mathbf{3 i}$ \\
$\mathbf{3 j}$ \\
$\mathbf{3 k}$ \\
$\mathbf{3 l}$ \\
$\mathbf{3 l}$ \\
$\mathbf{3 l}$ \\
\hline 1 \\
\hline-1 \\
\hline OTBS \\
\hline 1 \\
\hline
\end{tabular}

3d

Yield (\%)

er

$\begin{array}{ll}76 & 72: 28 \\ 75 & 77 \cdot 23 \\ 49 & 73: 27\end{array}$

49

73:27

82:18

$84: 16$

79:21

83:17

46:54

66:34

-

$-$

84:16

74:26

52:48

-

90.5:9.5

85:15

87.5:12.5

59:41

87:13

88.5:11.5

91:9

93:7

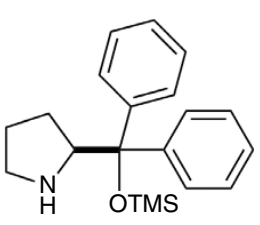

3a

$43 \%$ yield, $90.5: 9.5$

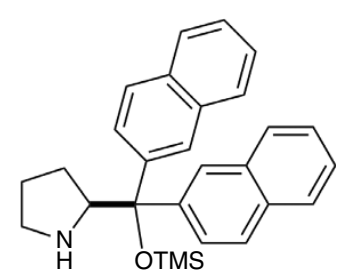

$3 \mathrm{~h}$<smiles>CCC[C@@H]1CC[C@@H](c2ccccc2)N1</smiles><smiles>CN1C(=O)[C@@H](Br)NC1(C)C</smiles>

$3 e$ $3 f$<smiles>COC(c1cc(C(F)(F)F)cc(C(F)(F)F)c1)(c1cc(C(F)(F)F)cc(C(F)(F)F)c1)C1C[C@@H]2CCCC[C@H]2N1</smiles>

3i

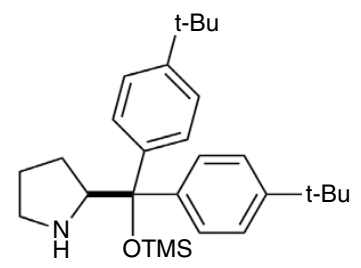

3k

DCM, dichloromethane; DIH, 1,3-diiodo-5,5-dimethylhydantoin; NIS, N-iodosuccinimide; TBS, tert-butyldimethylsilyl; TES, triethylsilyl; THF, tetrahydrofuran

${ }^{\star}$ Reactions were carried out in $1 \mathrm{ml}$ solvent at room temperature for $24 \mathrm{~h}$ using $0.1 \mathrm{mmol} \mathbf{1 a}, 0.1 \mathrm{mmol} \mathrm{I}^{+}$reagent, $0.1 \mathrm{mmol}$ amine $\mathbf{3}$ and $0.1 \mathrm{mmol} p \mathrm{NBA}$. Yield was determined by ${ }^{1} \mathrm{H} \mathrm{NMR}$ integration of the product against a standard. Er was determined by chiral HPLC. 
Table 2 | Substrate scope for the $\alpha$-cyclopropanation reaction ${ }^{\mathrm{a}, \mathrm{b}}$.

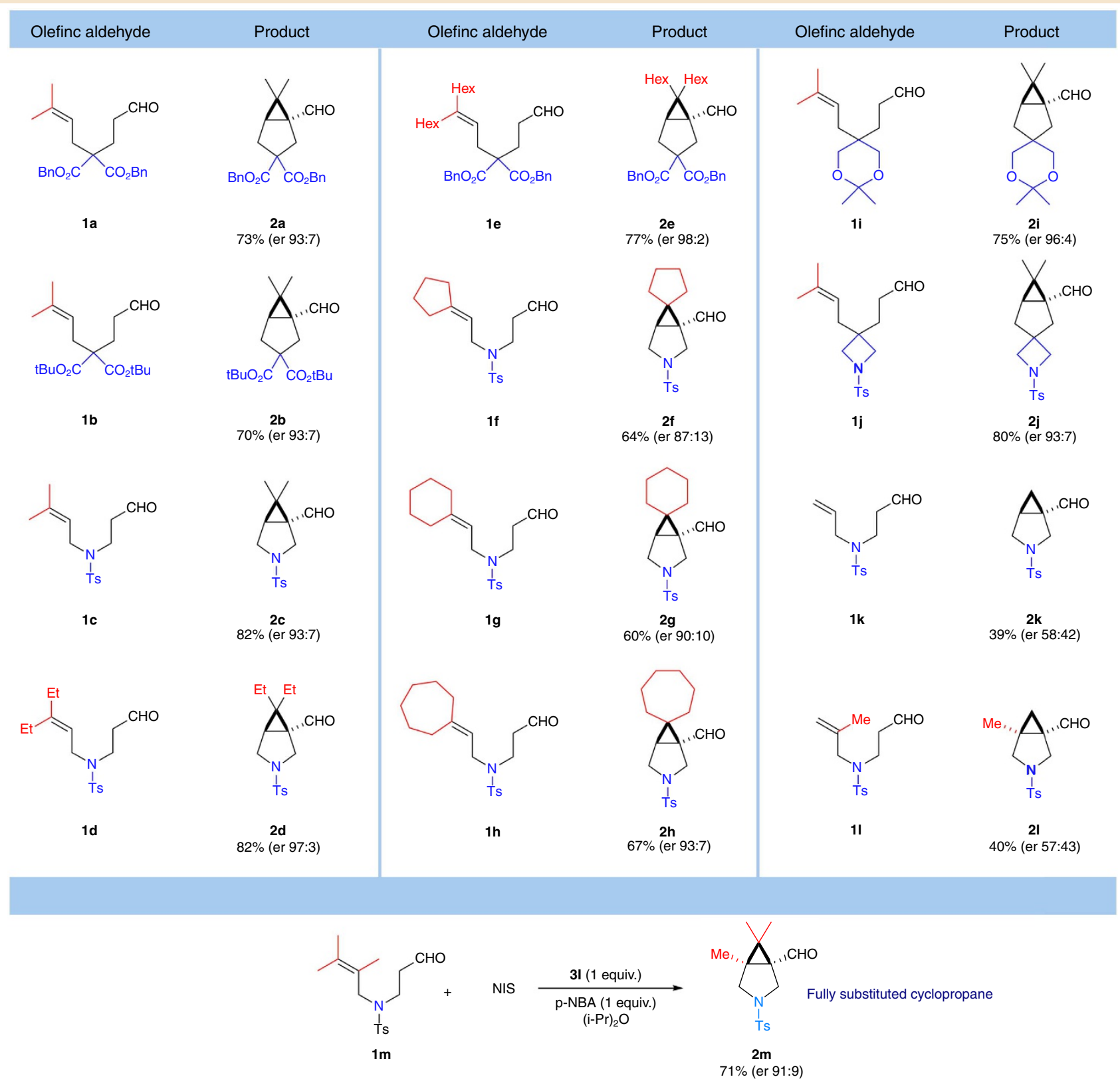

(a) Isolated yield. Er was determined by chiral HPLC. (b) Single diastereomer was observed for all products.

reaction proceeded with equal efficiency in dark and in the absence of any oxidant, suggesting a radical intermediate was unlikely. At this moment, we believe the reaction mechanism is in line with our original design (Fig. 2).

Rationale for the stereochemical control. The electrophilic alkylation is the stereoselectivity determining step, during which the $\alpha$-iodo iminium intermediate folds into a chair-like conformation. The double bond approaches the $\alpha$-carbon away from the bulky substituent of the amine and the olefin adopts a pseudo axial orientation in order to avoid steric crowding into the adjacent iminium moiety. This transition state model predicts the absolute stereochemistry of product to be $(1 R, 5 S)$, which was confirmed by X-ray crystallography of derivative 8c (Fig. 6, vide infra).

Synthetic manipulations of the products. The unique bridgehead aldehyde functionality enabled us to perform various chemical manipulations to access a number of chiral scaffolds (Fig. 7). $\mathrm{NaBH}_{4}$ reduction of $\mathbf{2 b}$ gave the corresponding alcohol $\mathbf{5 b}$ in $95 \%$ yield. Reductive amination using morpholine afforded amine $\mathbf{6 b}$ in $91 \%$ yield ${ }^{58}$. Horner-Wadsworth-Emmons reaction of $2 c$ installed an $\alpha, \beta$-unsaturated ester at the bridgehead ${ }^{59}$. Seyferth-Gilbert homolygation using the modified Bestmann's condition generated an alkyne functionality at the [3.1.0] bridgehead $^{60}$. Epoxidation using trimethyl sulfonium iodide 
a

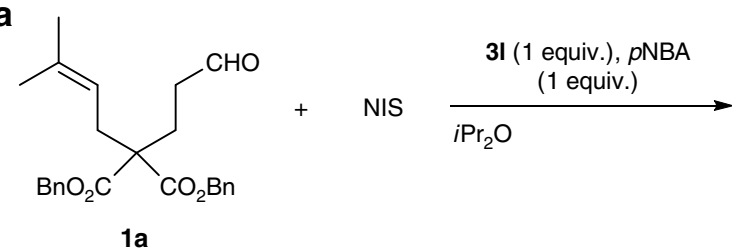

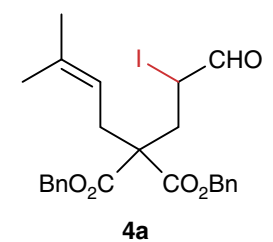

$5 \mathrm{~min}, 100 \%$ yield, er $=61: 39$

$40 \mathrm{~min}, 90 \%$ yield, er $=50: 50$

b

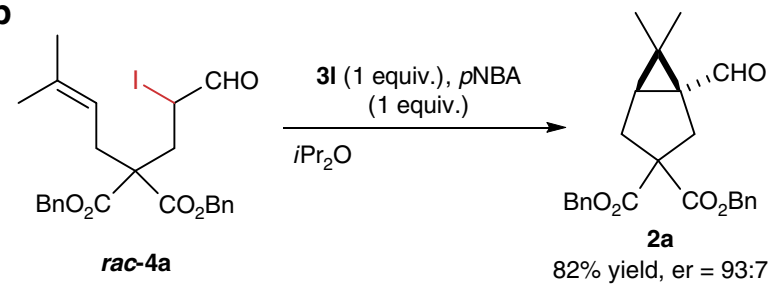

Figure 4 | Evidence of $\alpha$-iodo aldehyde as the key intermediate. (a) Under the standard condition for the $\alpha$-cyclopropanation, $\alpha$-iodo aldehyde $4 a$ was isolated after 5 and $40 \mathrm{~min}$. The product was found in low optical purity and quickly racemized during the reaction. (b) When racemic $\mathbf{4 a}$ was subjected to amine $\mathbf{3} \mathbf{l}$ in the absence of NIS, product $\mathbf{2 a}$ was obtained in good yield and er, indicating an efficient dynamic kinetic resolution process.

a<smiles>CC=CCN([12CH3])CCCO</smiles>

1n<smiles>C/C(=C\CN([12CH3])CCC=O)c1ccccc1</smiles>

10<smiles>C[C@@H]1CN([13CH3])C[C@H](C=O)[C@@H]1C</smiles>

2n

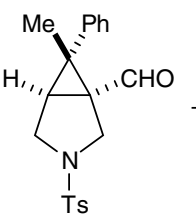

20<smiles>C[C@@H]1CN([125I])C[C@H](C=O)[C@@H]1C</smiles>

$2 n^{\prime}$

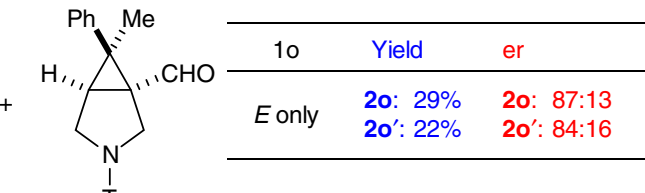

20

\begin{tabular}{cll}
\hline 1n & Yield & er \\
\hline$E: Z=5: 1$ & 2n: $48 \%$ & 2n: $77: 23$ \\
& 2n': $12 \%$ & 2n': 80:20 \\
\hline$E: Z=1: 3$ & 2n: $37 \%$ & 2n: $87: 13$ \\
& 2n': $16 \%$ & 2n': $84: 16$ \\
\hline
\end{tabular}

s

(1)

Figure 5 | Evidence for the stepwise annulation mechanism. (a) When a 5:1 E/Z mixture of $\mathbf{1 n}$ was subjected to the $\alpha$-cyclopropanation, products $\mathbf{2 n}$ and $\mathbf{2 n}$ ' were obtained as a pair of diastereomers favouring $\mathbf{2 n}$. Similar diastereomeric ratio and er were observed for a mixture of 1:3 E/Z isomers. (b) Substrate $\mathbf{1 0}$ in pure $E$-form led to a diastereomeric mixture of $\mathbf{2 0}$ and $\mathbf{2} \mathbf{o}^{\prime}$ in nearly 1:1 ratio. The results in $\mathbf{a}$ and $\mathbf{b}$ indicate the formation of the two new carbon-carbon bonds is stepwise.

afforded $9 \mathrm{c}$ as a mixture of diastereomers ${ }^{61}$. The formyl group of $2 \mathrm{c}$ was smoothly converted to methyl using Huang's modification of Wolff-Kishner reduction ${ }^{62}$. Deformylation using $\mathrm{RhCl}_{3}$ resulted concurrent cyclopropane opening to give 3 -vinylpyrrolidine 11c in $61 \%$ yield. The aldehyde group was retained in an acid promoted cyclopropane fragmentation to afford 3-formyl-4-vinylpyrrolidine 12c. Interestingly, skipped diene 13b was formed via Grob fragmentation ${ }^{63}$ when we carried out sulfonylation on alcohol $\mathbf{5 b}$. It is noteworthy that most of these derivatives would be very difficult to prepare otherwise.

Preliminary result for amine catalysis. It was intriguing that this cyclopropanation required $100 \mathrm{~mol} \%$ of the chiral amine to achieve high conversion. Our earlier results showed that the $\alpha$-iodo aldehyde could be generated in quantitative yield using $20 \mathrm{~mol} \%$ amine. The lack of amine turnover in the annulation step was likely due to the generation of HI that interfered with the enamine/iminium equilibrium. Various quenching agents were used in an attempt to speed up catalyst regeneration. However, no improvement was observed using bases and silver salts. On the other hand, moderate turnover can be achieved using $p \mathrm{NBA}$ as buffer and substrate 1a in excess (Fig. 8). In-depth investigation on further improving catalyst turnover is underway and will be reported in due course.

\section{Discussion}

In summary, we developed the first example of asymmetric intramolecular $\alpha$-cyclopropanation of aldehydes using a chiral amine promoter. We discovered that the $\alpha$-iodo iminium functionality could serve as a donor/acceptor carbene mimetic to engage in an intramolecular annulation reaction with an olefin. This strategy enables direct access to privileged chiral bicyclo[3.1.0] hexane scaffolds. The formation of the threemember ring is believed to occur through a stepwise double electrophilic alkylation cascade. This reaction represents the first asymmetric double $\alpha$-alkylation of aldehydes. The synthetic utility of the ring-fused cyclopropane products is demonstrated by diversified chemical derivatization. We expect the use of $\alpha$-iodoaldehyde as a versatile donor/acceptor warhead will 


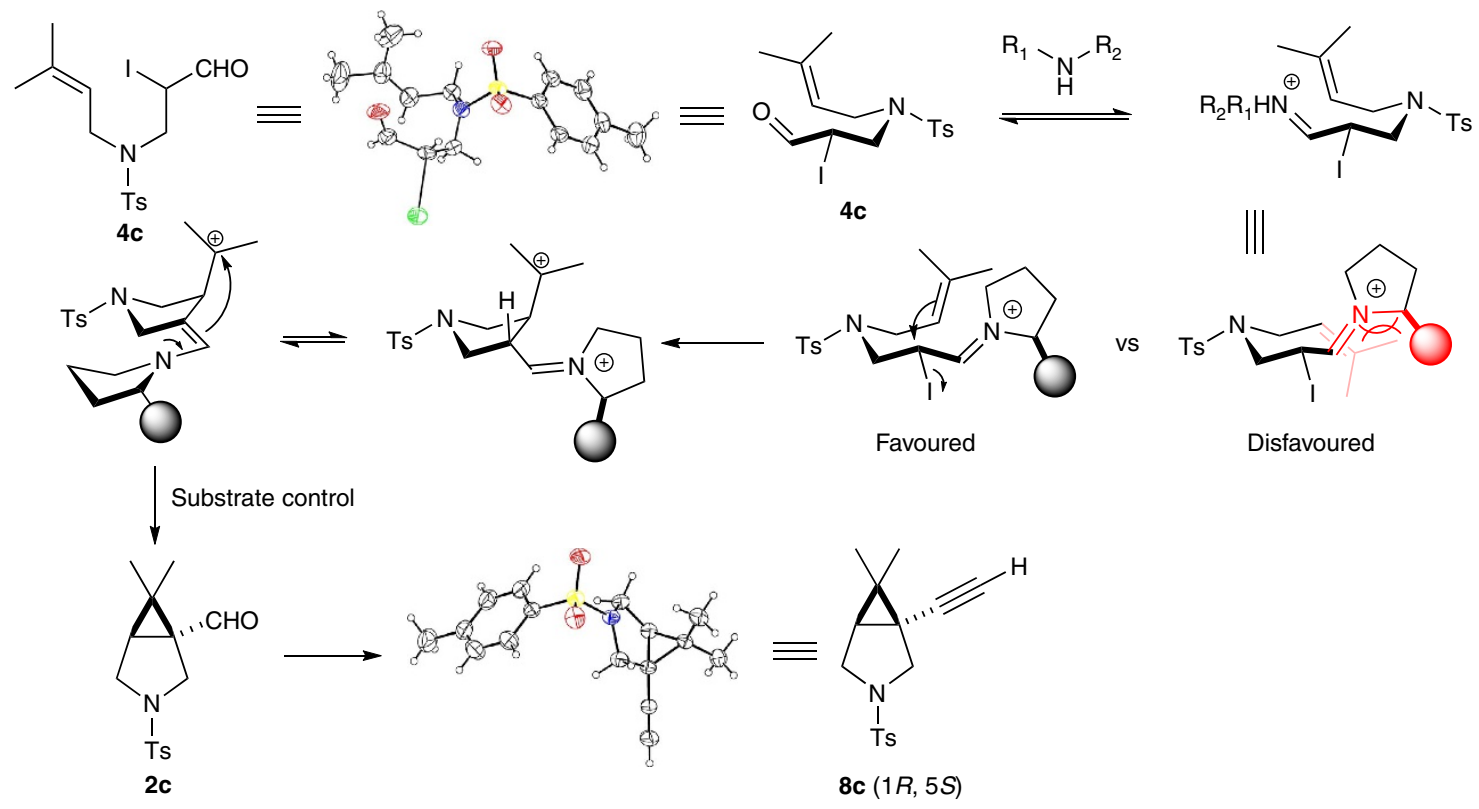

Figure 6 | Rationale for the stereoselectivity. There are four possible chair-like conformations for the first C-C bond formation. Owing to the chiral centre on the amine, only the two with the olefin moiety folded away from the bulky substituent are shown. The second C-C bond formation is substrate controlled as the [3.1.0]-bicyclic scaffold is much more stable in cis-form.

become a general approach for asymmetric di-functionalization of carbonyl compounds.

\section{Methods}

General methods and materials. Solvents for reactions were distilled according to general practice before use. All reagents were purchased and used without further purification unless specified otherwise. Amine catalyst was prepared based on the literature report ${ }^{64,65}$. Solvents for chromatography were technical grade and distilled before use. Flash chromatography was performed using 200-300 mesh silica gel with the indicated solvent system according to the standard techniques. Analytical thin-layer chromatography was performed using Huanghai silica gel plates with HSGF 254. Qingdao Haiyang Chemical HG/T2354-92 silica gel was used for silica gel flash chromatography. Visualization of the developed chromatogram was performed by ultraviolet absorbance $(254 \mathrm{~nm})$ or appropriate stains. ${ }^{1} \mathrm{H}$ NMR data were recorded on Bruker nuclear resonance spectrometers $(300,400$ or $500 \mathrm{MHz})$ unless specified otherwise. Chemical shifts $(\delta)$ in p.p.m. are reported as quoted relative to the residual signals of chloroform $\left({ }^{1} \mathrm{H}\right.$ 7.26 p.p.m. or ${ }^{13} \mathrm{C} 77.16$ p.p.m.). Multiplicities are described as: s (singlet), bs (broad singlet), $\mathrm{d}$ (doublet), t (triplet), q (quartet), m (multiplet); and coupling constants $(J$ ) are reported in Hertz $(\mathrm{Hz}),{ }^{13} \mathrm{C}$ NMR spectra were recorded on Bruker spectrometers $(75,101$ or $126 \mathrm{MHz})$ with total proton decoupling. High-resolution mass spectrometry (HRMS) (electrospray ionization (ESI)) analysis was performed by the Analytical Instrumentation Center at the Peking University, Shenzhen Graduate School, and HRMS data were reported with ion mass/charge $(\mathrm{m} / \mathrm{z})$ ratios as values in atomic mass units. Chiral HPLC was recorded on a Shimadzu LC-20A spectrometer using Daicel Chiralcel columns. ${ }^{1} \mathrm{H}$ NMR, ${ }^{13} \mathrm{C}$ NMR and HPLC spectra are provided for all products; see Supplementary Figs 1-106. For The Oak Ridge Thermal Ellipsoid Plot (ORTEP) structures of $\mathbf{2 b}$, rac-4c and $\mathbf{8 c}$, see Supplementary Figs 107-109. See Supplementary Methods for the characterization data for all compounds. See Supplementary Data 1-3 for X-ray CIF files of compounds 2b, rac-4c and 8c (CCDC 1405229, 1405230, 1405231).

Synthesis of substrates. Synthetic method A for substrates $1 a-1 b, 1 e$. Dibenzyl malonate was converted to dibenzyl 2-allyl-2-(3-methylbut-2-en-1-yl)malonate by double alkylation according to a similar reported procedure ${ }^{66}$. To a solution of this diene ( $5 \mathrm{mmol}, 1$ equiv.) in tetrahydrofuran (THF) $(50 \mathrm{ml})$ at $0^{\circ} \mathrm{C}$, 9-borabicyclo[3.3.1] nonane (1.5 equiv., $0.5 \mathrm{M}$ in THF) was slowly added by syringe over $10 \mathrm{~min}$. The reaction was allowed to warm to room temperature and stirred for $3 \mathrm{~h}$. Then $30 \mathrm{ml}$ of water and sodium perborate tetrahydrate $(4.5 \mathrm{~g})$ was added slowly (exothermic). The white suspension was stirred overnight. The mixture was filtered to remove the white solid. The filtrate was poured into a separatory funnel and the layers were separated. The aqueous phase was back-extracted three times with $50 \mathrm{ml}$ portions of ether. The combined organic layers were washed with $50 \mathrm{ml}$ of brine. The resulting solution was dried over sodium sulfate, filtered and concentrated to yield a clear oil, which was further purified by flash column chromatography to provide dibenzyl 2-(3-hydroxypropyl)-2-(3-methylbut-2-en-1$\mathrm{yl}$ )malonate. To a flask containing the solution of this primary alcohol in dichloromethane was added pyridinium chlorochromate (1.5 equiv.) and the reaction stirred at room temperature until all the starting material was consumed. Filter to remove the inorganic impurity and the product was isolated by flash column chromatography (ethyl acetate and petrol ether as eluent).

Synthetic method B for substrates 1c-1d, 1f-1h, 1k-10. To a solution of N-(3hydroxypropyl)-4-methylbenzenesulfonamide $(5 \mathrm{mmol})$ in dimethylformamide (DMF) $(40 \mathrm{ml})$, sodium hydride (60\% dispersion in mineral oil, 1.2 equiv.) was slowly added. The bubbling solution was stirred for $60 \mathrm{~min}$ before 3,3-dimethylallyl bromide (1.3 equiv.) was added (in some cases, heating is required). The reaction was stirred for $16 \mathrm{~h}$ before being partitioned between $\mathrm{H}_{2} \mathrm{O}$ and diethyl ether. The organic layer was washed with $\mathrm{H}_{2} \mathrm{O}(2 \times 40 \mathrm{ml})$ and brine $(40 \mathrm{ml})$, dried over sodium sulfate, filtered, concentrated and column chromatography can afford the precursor. The final product was obtained by pyridinium chlorochromate (PCC) oxidation.

Synthetic method C for substrate 1i. Compound dibenzyl 2-allyl-2-(3-methylbut-2-en-1-yl)malonate was reduced to 2-allyl-2-(3-methylbut-2-en-1-yl)propane1,3-diol with lithium aluminium hydride. To the solution of this diol (5 mmol, 1 equiv.) in THF $(100 \mathrm{ml}), 2,2$-dimethoxypropane $(6 \mathrm{mmol}, 1.2$ equiv.) and PTSA monohydrate $(50 \mathrm{mg}, 0.25 \mathrm{mmol})$ were added, the resulting solution was stirred overnight at room temperature. The solvent was removed and the residue was purified by silica gel chromatography to yield 5-allyl-2,2-dimethyl-5-(3-methylbut-2-en-1-yl)-1,3-dioxane. This compound subsequently underwent hydroboration/oxidation and subsequent PCC oxidation to afford the desired product (similar procedure as described above).

Synthetic method D for substrate $\mathbf{1 j}$. To a cooled $\left(-20^{\circ} \mathrm{C}\right)$ solution of 2-allyl-2(3-methylbut-2-en-1-yl)propane-1,3-diol ( $5 \mathrm{mmol}, 1$ equiv.) in dichloromethane $(50 \mathrm{ml})$, triethylamine $(20 \mathrm{mmol}, 4$ equiv.) and methanesulfonyl chloride $(10 \mathrm{mmol}$, 2 equiv.) were sequentially added. The mixture was stirred for $30 \mathrm{~min}$, then warmed to ambient temperature and partitioned between ethyl acetate and $1 \mathrm{~N} \mathrm{HCl}$. The organic layer was washed with brine, dried over $\mathrm{MgSO}_{4}$ and concentrated in vacuo to provide 2-allyl-2-(3-methylbut-2-en-1-yl)propane-1,3-diyl dimethanesulfonate. The solution of the resulting dimesylate, potassium carbonate $(15 \mathrm{mmol}, 3$ equiv.) and tosyl amide ( $7.5 \mathrm{mmol}, 1.5$ equiv.) in dimethylsulphoxide $(50 \mathrm{ml})$ was heated at $90{ }^{\circ} \mathrm{C}$ for $30 \mathrm{~h}$. When cooled to room temperature, water $(50 \mathrm{ml})$ was added, and the product was extracted with dichloromethane $(3 \times 50 \mathrm{ml})$. The combined organic layer was washed with brine $(3 \times 30 \mathrm{ml})$. Dried over sodium sulfate, filtered, concentrated and purified by column chromatography to deliver 3-allyl-3(3-methylbut-2-en-1-yl)-1-tosylazetidine. The final product $\mathbf{1 j}$ was obtained by sequential hydroboration/oxidation and subsequent PCC oxidation (similar procedure as described above). 
a<smiles>CCOC(=O)C1(C(=O)OCC)CC2C(C)(C)[C@]21CO</smiles>

5b, $95 \%$

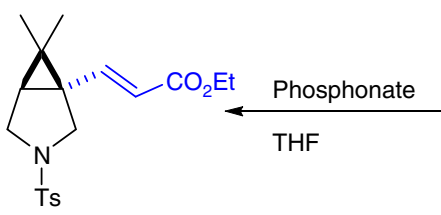

7c, $89 \%$<smiles>[3H]N1C[C@@H]2C(C)(C)[C@]2(C2CO2)C1</smiles>

9c, $87 \%$ dr: 1.6:1<smiles>C=C(C)[C@@H]1CCN([125I])C1</smiles>

11c, $61 \%$

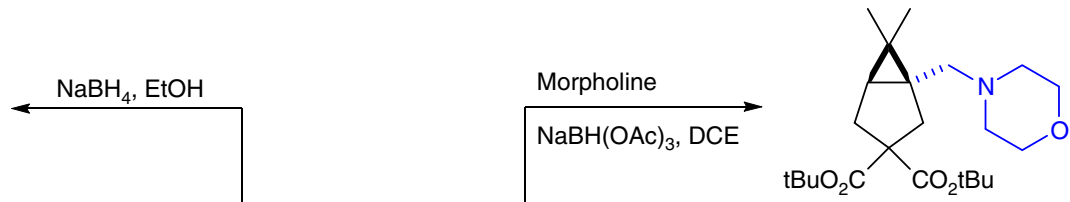

6b, $91 \%$

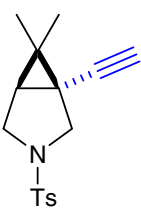

8c, $94 \%$

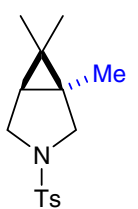

$10 c, 93 \%$

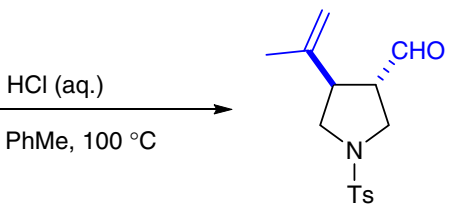

12c, $47 \%$<smiles>CCCCC(C)(C)C1(C(=O)OCC)CC2C(C)(C)[C@@]2(CO)C1</smiles>

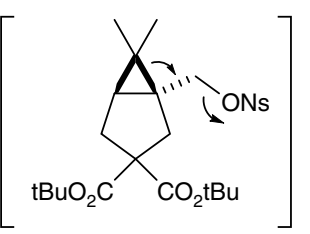<smiles>C=C(C)[C@H]1CC(CC(C)C)(C(=O)OCC)CC1=C</smiles>

13b, $60 \%$

Figure 7 | Chemical manipulation of the [3.1.0]-bicyclic aldehyde products. (a) The bridgehead formal group can be converted into a number of functionalities. (b) Transforming the free $\mathrm{OH}$ of $\mathbf{5 b}$ into a good leaving group prompts Grob fragmentation to form a skipped diene. Bestmann reagent, dimethyl (acetyldiazomethyl)phosphonate; DCE, dichloroethane; DEG, diethylene glycol; DME, diglyme; DMSO, dimethyl sulphoxide; dppp, 1,3bis(diphenylphosphino)propane; $\mathrm{NsCl}$, 4-nitrobenzenesulfonyl chloride; THF, tetrahydrofuran.<smiles>CC(C)=CCC(CCC=O)(OCc1ccccc1)C(=O)OCc1ccccc1</smiles>

1a, 2 equiv.

$$
+\quad \text { NIS } \frac{31(20 \text { mol\%) }}{\text { pNBA (1.0 equiv.), } i \mathrm{Pr}_{2} \mathrm{O}}
$$

1 equiv.

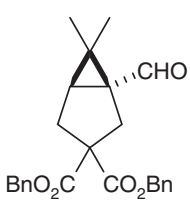

2a, $58 \%$, er $=91: 9$
Figure 8 | Catalytic turnover of the chiral amine. When 1a was used in excess, the reaction reached $58 \%$ isolated yield using 20 mol\% amine $\mathbf{3 1}$ reflecting a turnover number of 3 .

General procedure for $\alpha$-cyclopropanation of aldehydes. A 2-dram vial was charged with a solution of substrate $\mathbf{1}(0.1 \mathrm{mmol}, 1.0$ equiv.) in diisopropyl ether $(1 \mathrm{ml})$. Amine 31 ( $0.1 \mathrm{mmol}, 1.0$ equiv.) and $p \mathrm{NBA}(0.1 \mathrm{mmol}, 1.0$ equiv. $)$ were added sequentially. The reaction mixture was stirred for $5 \mathrm{~min}$ before NIS $(0.1 \mathrm{mmol}, 1.0$ equiv.) was added. The reaction vessel was capped with a screw cap and stirred for $24 \mathrm{~h}$ at $25^{\circ} \mathrm{C}$. The reaction mixture was directly subjected to silica gel flash column chromatography (ethyl acetate/petrol ether) to afford product 2. A racemic sample was prepared similarly using racemic amine.

\section{References}

1. Caine, D. Alkylsyions of Enols and Enolates in Comprehensive Organic Synthesis Vol. 3 (eds Trost, B. M. \& Fleming, I.) 1-63 (Pergamon, 1991).

2. Evans, D. A., Helmchen, G. \& Rüping, M. Chiral Auxiliaries in Asymmetric Synthesis In Asymmetric Synthesis - The Essentials. (eds Christmann, M. \& Bräse, S.) 3-9 (Wiley-VCH, 2007).

3. Gnas, Y. \& Glorius, F. Chiral auxiliaries - principles and recent applications. Synthesis 12, 1899-1930 (2006).

4. Myers, A. G. et al. Pseudoephedrine as a practical chiral auxiliary for the synthesis of highly enantiomerically enriched carboxylic acids, alcohols, aldehydes, and ketones. J. Am. Chem. Soc. 119, 6496-6511 (1997).

5. Doyle, A. G. \& Jacobsen, E. N. Enantioselective alkylations of tributyltin enolates catalyzed by $\mathrm{Cr}(\mathrm{salen}) \mathrm{Cl}$ : access to enantiomerically enriched allcarbon quaternary centers. J. Am. Chem. Soc. 127, 62-63 (2005).

6. Denmark, S. E., Gould, N. D. \& Wolf, L. M. A systematic investigation of quaternary ammonium ions as asymmetric phase-transfer catalysts. synthesis of catalyst libraries and evaluation of catalyst activity. J. Org. Chem. 76, 4260-4336 (2011).

7. Hashimoto, T. \& Maruoka, K. Recent development and application of chiral phase-transfer catalysts. Chem. Rev. 107, 5656-5682 (2007).

8. Vignola, N. \& List, B. Catalytic asymmetric intramolecular $\alpha$-alkylation of aldehydes. J. Am. Chem. Soc. 126, 450-451 (2004).

9. List, B. et al. The catalytic asymmetric $\alpha$-benzylation of aldehydes. Angew. Chem. Int. Ed. Engl 53, 282-285 (2014). 
10. Brown, A. R., Kuo, W.-H. \& Jacobsen, E. N. Enantioselective catalytic $\alpha$-alkylation of aldehydes via an SN1 pathway. J. Am. Chem. Soc. 132, 9286-9288 (2010).

11. Shaikh, R. R., Mazzanti, A., Petrini, M., Bartoli, G. \& Melchiorre, P. Prolinecatalyzed asymmetric formal $\alpha$-alkylation of aldehydes via vinylogous iminium ion intermediates generated from arylsulfonyl indoles. Angew. Chem. Int. Ed. Engl. 47, 8707-8710 (2010).

12. Caruana, L., Kniep, F., Johansen, T. K., Poulsen, P. H. \& Jørgensen, K. A. A New Organocatalytic Concept for Asymmetric $\alpha$-Alkylation of Aldehydes. J. Am. Chem. Soc. 136, 15929-15932 (2014).

13. Enders, D., Wang, C. \& Bats, J. W. Organocatalytic asymmetric domino reactions: a cascade consisting of a michael addition and an aldehyde $\alpha$-alkylation. Angew. Chem. Int. Ed. Engl. 47, 7539-7542 (2008).

14. Gualandi, A., Emer, E., Capdevila, M. G. \& Cozzi, P. G. Highly enantioselective $\alpha$ Alkylation of aldehydes with 1,3-benzodithiolylium tetrafluoroborate: a formal organocatalytic $\alpha$ Alkylation of aldehydes by the carbenium ion. Angew. Chem. Int. Ed. Engl. 50, 7842-7846 (2011).

15. Jiménez, J. et al. Enantio- and diastereoselective organocatalytic $\alpha$-alkylation of aldehydes with 3-substituted 2-(bromomethyl)acrylates. J. Org. Chem. 77, 747-753 (2012).

16. Ibrahem, I. et al. One-pot organocatalytic domino michael $/ \alpha$-alkylation reactions: direct catalytic enantioselective cyclopropanation and cyclopentanation reactions. Chem. Eur. J 14, 7867-7879 (2008).

17. Ibrahem, I. \& Córdova, A. Direct catalytic intermolecular $\alpha$-allylic alkylation of aldehydes by combination of transition-metal and organocatalysis. Angew. Chem. Int. Ed. Engl. 45, 1952-1956 (2006).

18. Hodgson, D. M., Charlton, A., Paton, R. S. \& Thompson, A. L. C-Alkylation of chiral tropane- and homotropane-derived enamines. J. Org. Chem. 78, 1508-1518 (2013).

19. Silvi, M., Arceo, E., Jurberg, I. D., Cassani, C. \& Melchiorre, P. Enantioselective organocatalytic alkylation of aldehydes and enals driven by the direct photoexcitation of enamines. J. Am. Chem. Soc. 137, 6120-6123 (2015).

20. Wilger, D. J., Grandjean, J. M., Lammert, T. \& Nicewicz, D. A. The direct anti-Markovnikov addition of mineral acids to styrenes. Nat. Chem 6, 720-726 (2014).

21. Beeson, T. D., Mastracchio, A., Hong, J.-B., Ashton, K. \& MacMillan, D. W. C. Enantioselective organocatalysis using SOMO activation. Science 316, 582-585 (2007).

22. Salaün, J. Cyclopropane derivatives and their diverse biological activities. Top. Curr. Chem. 207, 1-67 (2000).

23. Carson, C. A. \& Kerr, M. A. Heterocycles from cyclopropanes: applications in natural product synthesis. Chem. Soc. Rev. 38, 3051-3060 (2009).

24. Davies, H. M. L. \& Denton, J. R. Application of donor/acceptor-carbenoids to the synthesis of natural products. Chem. Soc. Rev. 38, 3061-3071 (2009).

25. Tang, P. \& Qin, Y. Recent applications of cyclopropane-based strategies to natural product synthesis. Synthesis 44, 2969-2984 (2012).

26. Bremeyer, N., Smith, S. C., Ley, S. V. \& Gaunt, M. J. An intramolecular organocatalytic cyclopropanation reaction. Angew. Chem. Int. Ed. Engl. 43, 2681-2684 (2004).

27. Johansson, C. C. C. et al. Enantioselective catalytic intramolecular cyclopropanation using modified cinchona alkaloid organocatalysts. Angew. Chem. Int. Ed. Engl. 45, 6024-6028 (2006).

28. Bull, J. A. \& Charette, A. B. Intramolecular Simmons - Smith cyclopropanation. studies into the reactivity of alkyl-substituted zinc carbenoids, effect of directing groups and synthesis of bicyclo[n.1.0]alkanes. $J$. Am. Chem. Soc. 132, 1895-1902 (2010).

29. Shen, J.-J. et al. Enantioselective iron-catalyzed intramolecular cyclopropanation reactions. Angew. Chem. Int. Ed. Engl. 53, 13188-13191 (2014).

30. Mamane, V., Gress, T., Krause, H. \& Fürstner, A. Platinum- and gold-catalyzed cycloisomerization reactions of hydroxylated enynes. J. Am. Chem. Soc. 126, 8654-8655 (2004).

31. Harrak, Y. et al. PtCl2-catalyzed cycloisomerizations of 5-en-1-yn-3-ol systems. J. Am. Chem. Soc. 126, 8656-8657 (2004).

32. Luzung, M. R., Markham, J. P. \& Toste, F. D. Catalaytic isomerization of 1,5enynes to bicyclo[3.1.0] hexenes. J. Am. Chem. Soc. 126, 10858-10859 (2004).

33. Buzas, A. \& Gagosz, F. Gold(I) catalyzed isomerization of 5-en-2-yn-1-yl acetates: an efficient access to acetoxy bicyclo[3.1.0]hexenes and 2-cycloalken1-ones. J. Am. Chem. Soc. 128, 12614-12615 (2006).

34. Toh, K. K., Wang, Y.-F., Ng, E. P. J. \& Chiba, S. Copper-mediated aerobic synthesis of 3-Azabicyclo[3.1.0]hex-2-enes and 4-Carbonylpyrroles from N-allyl/propargyl enamine carboxylates. J. Am. Chem. Soc. 133, 13942-13945 (2011).

35. Tong, X., Beller, M. \& Tse, M. K. A palladium-catalyzed cyclization - oxidation sequence: synthesis of Bicyclo[3.1.0] hexanes and evidence for SN2 C - O bond formation. J. Am. Chem. Soc. 129, 4906-4907 (2007).
36. Welbes, L. L., Lyons, T. W., Cychosz, K. A. \& Sanford, M. S. Synthesis of cyclopropanes via Pd(II/IV)-catalyzed reactions of Enynes. J. Am. Chem. Soc. 129, 5836-5837 (2007)

37. Tsujihara, T., Takenaka, K., Onitsuka, K., Hatanaka, M. \& Sasai, H. Pd ${ }^{\mathrm{II}} / \mathrm{Pd}^{\mathrm{IV}}$ catalytic enantioselective synthesis of Bicyclo[3.1.0]hexanes via oxidative cyclization of Enynes. J. Am. Chem. Soc. 131, 3452-3453 (2009).

38. Masutomi, K., Noguchi, K. \& Tanaka, K. Enantioselective cycloisomerization of 1,6-Enynes to Bicyclo[3.1.0]hexanes catalyzed by rhodium and benzoic acid. J. Am. Chem. Soc. 136, 7627-7630 (2014).

39. List, B., Lerner, R. A. \& Barbas, III C. F. Proline-catalyzed direct asymmetric aldol reactions. J. Am. Chem. Soc. 122, 2395-2396 (2000).

40. Ahrendt, K. A., Borths, C. J. \& MacMillan, D. W. C. New strategies for organic catalysis: the first highly enantioselective organocatalytic diels - alder reaction. J. Am. Chem. Soc. 122, 4243-4244 (2000).

41. Comito, R. J., Finelli, F. G. \& MacMillan, D. W. C. New strategies for organic catalysis: enantioselective intramolecular aldehyde $\alpha$-alkylation with simple olefins: direct access to homo-ene products. J. Am. Chem. Soc. 135, 9358-9361 (2013).

42. Rios, R. et al. A simple organocatalytic enantioselective cyclopropanation of $\alpha, \beta$-unsaturated aldehydes. Adv. Synth. Catal. 349, 1028-1032 (2007).

43. Xie, H., Zu, L., Li, H., Wang, J. \& Wang, W. Organocatalytic enantioselective cascade michael-alkylation reactions: synthesis of chiral cyclopropanes and investigation of unexpected organocatalyzed stereoselective ring opening of cyclopropanes. J. Am. Chem. Soc. 129, 10886-10894 (2007).

44. Vesely, J., Zhao, G.-L., Bartoszewicz, A. \& Córdova, A. Organocatalytic asymmetric nitrocyclopropanation of $\alpha, \beta$-unsaturated aldehydes. Tetrahedon Lett 49, 4209-4212 (2008).

45. Kunz, R. K. \& MacMillan, D. W. C. Organocatalytic asymmetric nitrocyclopropanation of $\alpha, \beta$-unsaturated aldehydes. J. Am. Chem. Soc. 127, 3240-3241 (2005).

46. Wang, Z., Li, X. \& Huang, Y. Direct $\alpha$-vinylidenation of aldehydes and subsequent cascade: gold and amine catalysts work synergistically. Angew. Chem. Int. Ed. Engl. 52, 14219-14223 (2013).

47. Halland, N., Braunton, A., Bachmann, S., Marigo, M. \& Jørgensen, K. A. Direct organocatalytic asymmetric $\alpha$-chlorination of aldehydes. J. Am. Chem. Soc. 126, 4790-4791 (2004).

48. Brochu, M. P., Brown, S. P. \& MacMillan, D. W. C. Direct and enantioselective organocatalytic $\alpha$-chlorination of aldehydes. J. Am. Chem. Soc. 126, 4108-4109 (2004).

49. Steiner, D. D., Mase, N. \& Barbas, III C. F. Direct asymmetric $\alpha$-fluorination of aldehydes. Angew. Chem. Int. Ed. Engl. 44, 3706-3710 (2005).

50. Beeson, T. D. \& MacMillan, D. W. C. Enantioselective organocatalytic $\alpha$ fluorination of aldehydes. J. Am. Chem. Soc. 127, 8826-8828 (2005).

51. Enders, D. \& Hüttl, M. R. M. Direct organocatalytic $\alpha$-fluorination of aldehydes and ketones. Synlett 6, 991-993 (2005).

52. Marigo, M., Fielenbach, D., Braunton, A., Kjærsgaard, A. \& Jørgensen, K. A. Enantioselective formation of stereogenic carbon-fluorine centers by a simple catalytic method. Angew. Chem. Int. Ed. Engl. 44, 3703-3706 (2005).

53. Bertelsen, S. et al. Organocatalytic asymmetric $\alpha$-bromination of aldehydes and ketones. Chem. Commun. 4821-4823 (2005).

54. Kano, T., Ueda, M. \& Maruoka, K. Direct asymmetric iodination of aldehydes using an axially chiral bifunctional amino alcohol catalyst. J. Am. Chem. Soc. 130, 3728-3729 (2008).

55. Winter, P. et al. Transforming terpene-derived aldehydes into 1,2-epoxides via asymmetric $\alpha$-chlorination: subsequent epoxide opening with carbon nucleophiles. Chem. Commun. 47, 12200-12202 (2011).

56. Swatschek, J., Grothues, L., Bauer, J. O., Strohmann, C. \& Christmann, M. A formal, one-pot $\beta$-chlorination of primary alcohols and its utilization in the transformation of terpene feedstock and the synthesis of a c2-symmetrical terminal bis-epoxide. J. Org. Chem. 79, 976-983 (2014).

57. Franzén, J. et al. A general organocatalyst for direct $\alpha$-functionalization of aldehydes: stereoselective $\mathrm{C}-\mathrm{C}, \mathrm{C}-\mathrm{N}, \mathrm{C}-\mathrm{F}, \mathrm{C}-\mathrm{Br}$, and $\mathrm{C}-\mathrm{S}$ bondforming reactions. scope and mechanistic insights. J. Am. Chem. Soc. 127, 18296-18304 (2005).

58. Abdel-Magid, A. F., Carson, K. G., Harris, B. D., Maryanoff, C. A. \& Shah, R. D. Reductive amination of aldehydes and ketones with sodium triacetoxyborohydride. studies on direct and indirect reductive amination procedures1. J. Org. Chem. 61, 3849-3862 (1996).

59. Wadsworth, W. S. \& Emmons, Jr W. D. The utility of phosphonate carbanions in olefin synthesis. J. Am. Chem. Soc. 83, 1733-1738 (1961).

60. Roth, G., Liepold, B., Müller, S. \& Bestmann, H. J. Further improvements of the synthesis of alkynes from aldehydes. Synthesis 1, 59-62 (2004).

61. Corey, E. J. \& Chaykovsky, M. Dimethyloxosulfonium methylide ((CH3)2SOCH2) and dimethylsulfonium methylide ((CH3)2SCH2). Formation and application to organic synthesis. J. Am. Chem. Soc. 87, 1353-1364 (1965).

62. Huang, M. A simple modification of the Wolff-Kishner reduction. J. Am. Chem. Soc. 68, 2487-2488 (1946). 
63. Grob, C. A. \& Baumann, W. Die 1,4-Eliminierung unter Fragmentierung. Helv. Chim. Acta. 38, 594-610 (1955).

64. Kemppainen, E. K., Sahoo, G., Valkonen, A. \& Pihko, P. M. MukaiyamaMichael reactions with acrolein and methacrolein: a catalytic enantioselective synthesis of the C17-C28 fragment of pectenotoxins. Org. Lett. 14, 1086-1089 (2012).

65. Liu, Y.-K., Ma, C., Jiang, K., Liu, T.-Y. \& Chen, Y.-C. Asymmetric tandem michael addition - wittig reaction to cyclohexenone annulation. Org. Lett. 11, 2848-2851 (2009)

66. Marigo, M., Wabnitz, T. C., Fielenbach, D. \& Jørgensen, K. A. Enantioselective organocatalyzed $\alpha$ sulfenylation of aldehydes. Angew. Chem. Int. Ed. Engl. 44, 794-797 (2005)

\section{Acknowledgements}

This work is financially supported by the National Natural Science Foundation of China (21372013 and 21572004) and the Shenzhen Peacock Program (KQTD201103). Y.H. thanks the Ministry of Education (MOE) for the Program for New Century Excellent Talents in University. The Shenzhen municipal development and reform commission is thanked for their generous support of a public service platform programme. We thank Professor Benjamin List for insightful suggestions.

\section{Author contributions}

Y.H. conceived and directed the project. C.L. and Z.W. performed the experiments. Y.H. and C.L. analysed the data and wrote the manuscript.

\section{Additional information}

Accession Codes: The X-ray crystallographic coordinates for structures reported in this study have been deposited at the Cambridge Crystallographic Data Centre (CCDC), under deposition numbers CCDC 1405229, 1405230, 1405231. These data can be obtained free of charge from the Cambridge Crystallographic Data Centre via www.ccdc.cam.ac.uk/data_request/cif.

Supplementary Information accompanies this paper at http://www.nature.com/ naturecommunications

Competing financial interests: The authors declare no competing financial interests.

Reprints and permission information is available online at http://npg.nature.com/ reprintsandpermissions/

How to cite this article: Luo, C. et al. Asymmetric intramolecular $\alpha$-cyclopropanation of aldehydes using a donor/acceptor carbene mimetic. Nat. Commun. 6:10041 doi: $10.1038 /$ ncomms10041 (2015).

\section{(c) (i)}

This work is licensed under a Creative Commons Attribution 4.0 International License. The images or other third party material in this article are included in the article's Creative Commons license, unless indicated otherwise in the credit line; if the material is not included under the Creative Commons license, users will need to obtain permission from the license holder to reproduce the material. To view a copy of this license, visit http://creativecommons.org/licenses/by/4.0/ 